\title{
Endovascular administration of magnetized nanocarriers targeting brain delivery after stroke.
}

\begin{abstract}
Alba Graystona, Yajie Zhang ${ }^{\mathrm{b}}$, Miguel Garcia-Gabilondo ${ }^{\mathrm{a}}$, Mercedes Arrúe ${ }^{\mathrm{a}}$, Abraham Martin, Peter Kopcanskye, Milan Timko ${ }^{e}$, Jozef Kovace ${ }^{e}$ Oliver Strbak ${ }^{f}$, Laura Castelloteg, Sara Bellolih,i, Rosa M. Moresco ${ }^{h, j}$, Maria Picchio ${ }^{h, k}$, Anna Roig ${ }^{b,{ }^{*}}$, Anna Rosella, ${ }^{a}$.

aNeurovascular Research Laboratory, Vall d'Hebron Institut de Recerca, Universitat Autònoma de Barcelona (VHIR-UAB), Passeig Vall d'Hebron 119-129, 08035 Barcelona, Catalonia, Spain; bInstitut de Ciència de Materials de Barcelona (ICMAB-CSIC), Campus UAB, 08193 Bellaterra, Catalonia, Spain; 'Achucarro Basque Center for Neuroscience, Laboratory of Neuroimaging and biomarkers of inflammation, E-48940 Leioa, Spain; d/kerbasque Basque Foundation for Science, 48013 Bilbao, Spain; eInstitute of Experimental physics, SAS, 04001 Kosice, Slovakia; 'Biomedical Center Martin, Jessenius Faculty of Medicine in Martin, Comenius University in Bratislava, 03601 Martin, Slovakia; 9Department of Clinical Biochemistry, Clinical Laboratories, Vall d'Hebron

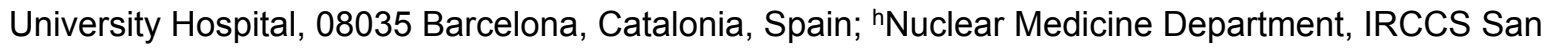
Raffaele Scientific Institute, 20132 Milan, Italy; institute of Molecular Bioimaging and Physiology (IBFM) of CNR, 20090 Segrate, Italy; 'Department of Medicine and Surgery, University of Milano Bicocca, 20126 Milan, Italy; kVita-Salute San Raffaele University, 20132 Milan, Italy.
\end{abstract}

${ }^{*}$ Corresponding Authors: Anna Rosell: Neurovascular Research Laboratory Vall d'Hebron Research Institute (VHIR). Passeig Vall d'Hebron 119-129; 08035, Barcelona, Catalonia, Spain. Tel. +34 934894029; anna.rosell@vhir.org. And Anna Roig: Institut de Ciència de Materials de Barcelona (ICMAB-CSIC), Campus UAB, 08193 Bellaterra, Catalonia, Spain; roig@icmab.es. 


\title{
Running Head: Improved brain targeting of nanocarriers in stroke
}

\begin{abstract}
The increasing use of mechanical thrombectomy in stroke management has opened the window to local intraarterial brain delivery of therapeutic agents. In this context, the use of nanomedicine could further improve the delivery of new treatments with specific brain targeting, tracking and guidance. In this study we take advantage of this new endovascular approach to deliver biocompatible poly(DL-lactic-co-glycolic acid) (PLGA) nanocapsules functionalized with superparamagnetic iron oxide nanoparticles and Cy7.5 for magnetic targeting, magnetic resonance and fluorescent molecular imaging. A complete biodistribution study in naïve $(n=59)$ and ischemic $(n=51)$ mice receiving intravenous or intraarterial nanocapsules, with two different magnet devices and imaged from 30 min to $48 \mathrm{~h}$, showed an extraordinary advantage of the intraarterial route for brain delivery with a specific improvement in cortical targeting when using a magnetic device in both control and ischemic conditions. Safety was evaluated in ischemic mice $(n=69)$ showing no signs of systemic toxicity nor increasing mortality, infarct lesions or hemorrhages. In conclusion, the challenging brain delivery of therapeutic nanomaterials could be efficiently and safely overcome with a controlled endovascular delivery and magnetic targeting, which could be considered in the context of endovascular interventions delivering multiple treatments for stroke.
\end{abstract}

Keywords: stroke, endovascular treatment, PLGA, nanocarrier, magnetic targeting 


\section{Introduction}

Given the narrow time-window and insufficient performance of thrombolytic therapies in large vessel occlusion strokes, endovascular therapy (EVT) has been in the spotlight of stroke management over the past years. ${ }^{1}$ EVT is now increasingly used throughout developed countries, with substantially improved devices showing successful recanalization rates within the first $6 \mathrm{~h}$ after symptoms onset. $^{2-6}$ Furthermore, the current American Stroke Association/American Heart Association guidelines have extended this time window up to $24 \mathrm{~h}$ in patients that meet specific eligibility criteria as demonstrated in the DAWN or DEFUSE clinical trials. ${ }^{7-9}$ However, despite these enormous advances in stroke management, the stroke community is still investigating on new treatments to improve functional recovery beyond the acute phase, when rehabilitation therapies are the only approved treatments. ${ }^{10}$ In this context, the extensive use of EVT in the daily clinical practice has also opened the opportunity for direct deliveries to the brain vasculature, through which neuroprotective or neurorestorative treatments could be directly administered in the ischemic hemisphere thus helping to overcome a significant drawback of systemic drug delivery. ${ }^{11,12}$ In this regard this administration route has already been used in stroke clinical trials to administer autologous stem cells, ${ }^{13}$ drugs such as verapamil ${ }^{14}$ or to induce cerebral hypothermia by administering cold isotonic saline..$^{15}$

In this scenario nanomedicine-based strategies are optimal candidates to synergistically exploit the advantages of endovascular approaches by tracking the therapeutic agent with imaging tags, reducing drug doses with a sustained release or improving brain targeting with functionalized biomaterials. In this light, we aimed at studying for the first time the advantages of the post-stroke endovascular delivery of biocompatible nanomaterials combined with specific magnetic brain 
targeting providing a complete safety, feasibility and biodistribution study. For this we have chosen poly(D-L-lactic-co-glycolic acid) (PLGA) as an FDA-approved and biocompatible polymer that is currently used in over 10 therapeutic nanoformulations, with the potential to encapsulate a large variety of therapeutic agents and integrate different imaging moieties. ${ }^{16,17}$ For the present study, the PLGA nanocapsules (NC) were functionalized with superparamagnetic iron oxide nanoparticles (SPION) and Cy7.5 for the improved magnetic targeting and brain imaging.

Our data provides evidence of the potential use and advantages of the endovascular delivery of nanomaterials after cerebral ischemia, which could be considered in the context of EVT to deliver multiple therapeutic agents.

\section{Materials and Methods}

\section{Animals}

All procedures were approved by the Ethics Committee for Animal Experimentation of the Vall d'Hebron Research Institute and Universitat Autònoma de Barcelona (protocol numbers 70/18 and CEEA-4784) and conducted in compliance with the Spanish legislation and in accordance with the Directives of the European Union. A total of 129 C57BL/6JRj (C57) and 88 Balbc AnNRj (Balbc) adult male mice $(23.7 \pm 1.5 \mathrm{~g}$ and $23.8 \pm 1.2 \mathrm{~g}$, respectively, 7 to 9 weeks), purchased from Janvier laboratories (Saint Berthevin, France), were used in this study. Mice were housed in groups and kept in a climate-controlled environment on a $12 \mathrm{~h}$ light/dark cycle. Food and water were available ad libitum. At the end of the study, a total of 59 mice were excluded: 21 due to failure/complications of the experimental procedures, 34 due to mortality after the interventions and 4 according to the exclusion criteria of the experimental model. A total of 38 mice were used for background control 
during fluorescent molecular imaging (FMI) acquisitions. All in vivo experiments are reported according to the ARRIVE 2.0 guidelines. ${ }^{18}$ Complete schemes of the experimental designs are presented in Figures 1-4 and 6.

\section{Middle Cerebral Artery occlusion (MCAo)}

C57BL/6 mice were subjected to 60 min MCAo by introducing an intraluminal filament through the right internal carotid artery (ICA), as described elsewhere. ${ }^{19}$ All animals were anesthetized with isoflurane via facemask ( $5 \%$ for induction, $1.5 \%$ for maintenance in air, $79 \% \mathrm{~N}_{2}: 21 \% \mathrm{O}_{2}$ ). Body temperature was maintained at $37^{\circ} \mathrm{C}$ using a self-regulated heating pad connected to a rectal probe. Mice eyes were protected from corneal damage during surgery using an ophthalmic lubricating ointment (Lipolac ${ }^{\mathrm{TM}}$; Angelini Farmaceutica, Barcelona, Spain) and analgesia (subcutaneous buprenorphine $0.1 \mathrm{mg} / \mathrm{kg}$; Divasa Farma-Vic S.A., Barcelona, Spain) was given before starting the surgical procedure and daily during the first 5 days after the surgery to minimize their pain and discomfort. Additionally, $0.5 \mathrm{~mL}$ of saline was daily administered subcutaneously for post-surgical recovery during the first 5 days. Nutritionally fortified water gel (DietGel Recovery®, ClearH $2 \mathrm{O} \otimes$, Portland, ME, USA) was given to mice from Safety study 2 one week prior to surgery for habituation and was available ad libitum after surgery.

After surgical exposure of the right Common Carotid Artery (CCA) bifurcation into the External Carotid Artery (ECA) and the ICA, a silicone-coated nylon monofilament (Doccol Corporation, Sharon, MA, USA, reference number: 602256PK10Re) was introduced through the ECA and directed towards the ICA to occlude the MCA. Occlusion was monitored by laser Doppler flowmetry using a flexible fibreoptic probe (Moor Instruments, Devon, UK) placed on the surface of the distal MCA branch, and confirmed by the decrease of the registered cerebral blood flow (CBF) $>80 \%$ 
( $100 \%$ being considered as the pre-ischemia CBF value). After occlusion, animals woke up and were re-anesthetized for reperfusion $60 \mathrm{~min}$ later as described above by removing the monofilament. Only animals recovering $>80 \%$ of $\mathrm{CBF}$ after filament removal were finally included.

\section{Nanocarriers synthesis}

PLGA-NC were synthetized by a double emulsion solvent evaporation method and functionalized

with SPION and Cy7.5 (Figures $1(\mathrm{a})$ and $1(\mathrm{c})$ ), as described previously. ${ }^{20}$ Briefly, SPION were embedded in the PLGA shell by adding oleic acid-coated SPION with an average diameter of $9 \mathrm{~nm}$ in the PLGA organic phase during the first emulsification, and modified PLGA-Cy7.5 was also added in the organic phase in addition to the commercial PLGA. The obtained NCs suspension in trehalose aqueous solution was lyophilized and stored at $4^{\circ} \mathrm{C}$ with desiccant silica gel until each experimental use when they were resuspended in saline. The NC were freshly dispersed at the desired concentration before use, vortexed for $1 \mathrm{~min}$ and sonicated in an ultrasound bath for $3 \mathrm{~min}$ at $83.3 \mathrm{~W} / \mathrm{L}$ and $48 \mathrm{kHz}$. Details of the different $\mathrm{NC}$ batches used in this study are listed in the Supplementary Table S1.

\section{Administration routes}

Systemic intravenous administration through the tail vein and intraarterial administration through the ICA of the NC dispersed in saline (either 1.6 or $0.8 \mathrm{mg} /$ animal, corresponding to the biodistribution and therapeutic doses, respectively) were conducted; see Figure 1(a). The therapeutic dose corresponds to the approved doses of iron Feridex ${ }^{\circledR}($ Endorem $®) .{ }^{21}$ The surgical procedure for the intraarterial administration was protocolized in the intraluminal MCAo model by cannulating the ECA 30 min after reperfusion under anesthesia as described above, with a polyimide microcatheter (0.2$0.22 \mathrm{~mm}$ OD, 0.14-0.15 mm ID; MicroLumen, Oldsmar, FL) directed towards the ICA and connected 
to an infusion pump (PHD 2000 Advance Syringe Pump, Harvard Apparatus, Holliston, Massachusetts, US) at an infusion rate of $75 \mu \mathrm{L} / \mathrm{min}$, total volume $150 \mu \mathrm{L}$. Sham animals underwent the same surgical procedure as for the intraluminal MCAo model except that the microcatheter was introduced through the ECA without previously occluding the MCA. Note that the pterygopalatine artery was permanently sutured in all cases to avoid perfusion towards off-target areas. The cortical CBF was registered before, during and after intraarterial administration of the NC as described above and according to the experimental design in each group. On the other hand, for intravenous administrations a volume of $150 \mu \mathrm{L} \mathrm{NC}$ suspension was administered using a $25 \mathrm{G}$ needle through the lateral tail vein after vessel dilatation using a heat lamp.

\section{Magnet devices}

To study the advantages of an external magnetic field for specific brain targeting of the magnetized $\mathrm{NC}$, two different iron-neodymium-boron $(\mathrm{FeNdB})$ magnets were constructed considering the mouse brain anatomy: permanent Magnet 1 (M1) and focused Magnet 2 (M2), together with non-magnetic pieces serving as controls, differing on the final size: Fake 1 (F1) and Fake 2 (F2).

M1 consists of a circular permanent magnet of $5 \times 2 \mathrm{~mm}$ covered by a soft iron yoke and a biocompatible polymer support frame (final size of the device was $8 \times 3 \mathrm{~mm}$ ). M2 consists of a focused magnet with a $2 \times 1$ matrix of submagnets covered with nickel and a thin biocompatible polyurethane/epoxy film, with a final cylindrical shape of $6 \times 3 \mathrm{~mm}$. F1 and F2 were manufactured as M1 and M2, respectively, except for a brass core instead of FeNdB. The spatial distribution of the magnetic field and magnetic force of $\mathrm{M} 1$ and $\mathrm{M} 2$ was characterized with the standard QUICKFIELD software. The modelling was calculated in the plane $3 \mathrm{~mm}$ over the surface of the magnet devices (Figure 5). 
The magnetic field induction and magnetic field gradient of the magnet devices M1 and M2 was measured by 3D Hall probe. The magnetic force was calculated according to the distance from the center of the magnet at $3 \mathrm{~mm}$ in height from the magnet surface.

The magnet/fake device was implanted on the right (ipsilateral) hemisphere prior to the NC administration (under anesthesia, as described in the supplementary materials) attached to the skull with superglue. The skin was closed over the device using Histoacry|® (Figure 1(a)) and was removed before the first in vivo image acquisition.

\section{Biodistribution by FMI}

FMI was performed to characterize the Cy7.5_NC batch-fluorescence in vitro (for details, please see the supplementary materials) and to track the NC biodistribution in vivo and ex vivo in single organs using a Xenogen IVIS $®$ spectrum. Prior to in vivo administrations, the fluorescence of Cy7.5_NC batches was characterized by FMI. Briefly, a series of concentrations of Cy7.5-labeled NC in $100 \mu \mathrm{L}$ of saline were prepared for each batch in a 96-well plate and imaged using a

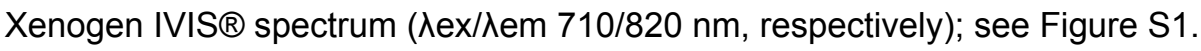

The general protocol for the in vivo biodistribution studies started with mice being anesthetized with isoflurane via facemask $\left(5 \%\right.$ for induction, $1.5 \%$ for maintenance in $\left.95 \% \mathrm{O}_{2}\right)$ and images being acquired at different time points post-injection according to each experimental design $(\lambda \mathrm{ex} / \mathrm{\lambda em}$ $710 / 820 \mathrm{~nm}$, respectively) in dorsal and ventral views of the whole body. Between image acquisitions mice recovered from anesthesia in temperature-controlled recovery cages. All animals were euthanized by cervical dislocation under anesthesia after the last in vivo acquisition and brain,

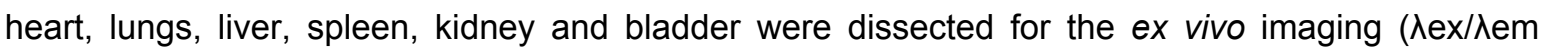


$745 / 820 \mathrm{~nm}$, respectively) in dorsal and ventral views. For details of the FMI analysis, please see the supplementary materials.

Biodistribution study 1: To first study biodistribution of the NC following systemic or endovascular routes and the influence of the first magnet device for brain targeting, randomly assigned naïve Balbc mice were injected either intravenously $(n=16)$ or intraarterially $(n=10)$ with the $1.6 \mathrm{mg}$ biodistribution dose of Cy7.5_NC following the aforementioned protocols. Each intravenous/intraarterial group was further randomly divided into the M1 and the control F1 groups. The fake/magnet devices were implanted for $30 \mathrm{~min}$ after the NC administration and FMI in vivo images were acquired at $30 \mathrm{~min}, 1 \mathrm{~h}$ and $3 \mathrm{~h}$ post-injection (Figures $1(\mathrm{a})$ and 2(a)). After the last acquisition, mice organs were obtained for ex vivo imaging as described above. Biodistribution study 2: to further investigate the influence of the magnetic field on the NC brain retention, naïve Balbc mice receiving the $1.6 \mathrm{mg}$ biodistribution dose of Cy7.5_NC intraarterially were randomly assigned into the M1 $(n=7)$ and the control F1 $(n=8)$ groups, implanted and administered as described above. A single image acquisition was conducted at $3 \mathrm{~h}$ post-injection when the brain was removed and divided into cortical and subcortical regions for the ex vivo acquisition (Figures $1(\mathrm{a})$ and 2(b)).

Biodistribution study 3: next, we studied the biodistribution of the NC after acute intraarterial administration in the MCAo model, with magnetic guidance by two magnet devices with different magnetic fields. A total of 16 C57 mice receiving the $0.8 \mathrm{mg}$ therapeutic dose of Cy7.5_NC intraarterially were randomly divided into the two magnet groups (M1 and M2) and a control fake F2 group. The magnet/fake devices were removed after $3 \mathrm{~h}$ before the first FMI acquisition time-point 
which was repeated at $48 \mathrm{~h}$ post-injection. After the last image acquisition organs were obtained for ex vivo imaging (Figures $1(\mathrm{a})$ and $5(\mathrm{a})$ ).

\section{Biodistribution by Magnetic Resonance Imaging (MRI)}

Biodistribution study 4: MRI was performed to further characterize the retention and spatial distribution of the NC in the brain after acute intraarterial administration in the MCAo model with magnetic guidance by the M2 device, which showed a better performance in the FMI Biodistribution study 3 (Figures 1(a) and 5(b)). A total of 17 C57 mice receiving the $0.8 \mathrm{mg}$ therapeutic dose of Cy7.5_NC through the ICA were randomly divided into the M2 and the control F2 groups, implanted and administered as in Biodistribution study 3. A single in vivo MRI was carried out at $48 \mathrm{~h}$ postinjection using a BioSpec 70/30 USR scanner with a 7 T horizontal magnet (Bruker BioSpin, Ettlingen, Germany) equipped with a $72 \mathrm{~mm}$ inner diameter linear volume coil as a transmitter and a mouse brain surface coil as a receiver. High-resolution T2-weighted and high-resolution T2*weighted images were acquired to visualize the ischemic lesion and the hypointensities representing the SPION-labelled NC, respectively. For details of the acquisition protocols and MRI analysis, please see the supplementary materials.

\section{Safety study in the MCAo model}

Safety study 1: to assess the safety of acute endovascular NC administration after cerebral ischemia, a total of 26 C57 ischemic mice were randomly assigned into 2 groups: the vehicle group receiving $150 \mu \mathrm{L}$ of saline $(n=13)$ with a fake magnet $(F 1)$, and the treatment group receiving the 0.8 mg therapeutic dose of $\mathrm{NC}$ in $150 \mu \mathrm{L}$ of saline $(n=13)$ with a magnet (M1), following the aforementioned protocols of pre-clinical stroke and endovascular administration through the ICA (Figures 1(a), 3(a) and 4(a)). Both groups were followed for $48 \mathrm{~h}$, when blood was collected from 
the right ventricle in EDTA tubes for biochemical analysis and animals were euthanized under deep anesthesia by cervical dislocation to dissect the brain for 2,3,5-triphenyltetrazolium chloride (TTC) staining and intracerebral hemorrhage evaluation.

Safety study 2: C57 ischemic mice $(\mathrm{n}=8)$ receiving the $0.8 \mathrm{mg}$ therapeutic dose of NC through the ICA were followed for 4 weeks when under deep anesthesia blood was collected from the right ventricle in EDTA tubes for biochemical analysis. Plasma control samples were also included from naïve C57 mice $(n=8)$ and ischemic C57 mice without treatment followed at $48 \mathrm{~h}(n=4)$ and at 4 weeks (n=8). (Figures $1(a)$ and $4(a))$.

\section{Prussian blue and vessel staining with lectin-FITC}

To confirm the presence of the NC in the brain, we performed specific staining for ferric iron as part of the SPION of the NC. Animals from Biodistribution study 4 were euthanized under deep anesthesia by exsanguination followed by transcardial perfusion with cold saline and the brain, liver and spleen were fixed with $10 \%$ formalin, paraffin-embedded and sliced in $5 \mu$ m-thick sections with a microtome. After deparaffinization and rehydration, the sections were stained with a Prussian blue iron stain kit (Polysciences Inc, USA) following the manufacturer's protocol except for that potassium ferrocyanide:hydrochloric acid mix solution was kept for a total of 40 min and nuclear Fast Red for 1 min. Images were acquired after dehydration and mounting with DPX medium (Ref. 06522, Sigma-Aldrich, St. Louis, USA) with a transmitted light microscope (Leica, Germany) and an automatic slide scanner (PANNORAMIC MIDI II, 3DHISTECH Ltd, Hungary).

To confirm the distribution of NC (Cy7.5-labeled) in brain microvessel structures as seen by Prussian blue staining, vasculature was stained in consecutive brain slices with lectin-FITC as follows: after deparaffinization and rehydration, the sections were incubated overnight with lectin- 
FITC from Lycopersicon esculentum (Ref. L0401, Sigma-Aldrich, Germany) diluted 1:200 in PBS1\% Tween20. After dehydration and mounting with antifade mounting medium (Vectashield, CA, USA), images were acquired with a confocal laser scanning microscope (LSM880, Zeiss, Germany).

\section{Infarct volume and intracerebral hemorrhage evaluation}

Ischemic C57 mice from Safety study 1 were euthanized at $48 \mathrm{~h}$ after administration postreperfusion by cervical dislocation. Brains were removed, sliced in $1 \mathrm{~mm}$ thick coronal sections and stained with TTC (Sigma, St.Louis, MO, USA) in saline at room temperature for $15 \mathrm{~min}$. Infarct volumes were quantified by a treatment-blinded researcher with the Image $\mathrm{J}$ software and corrected by edema as previously described. ${ }^{22}$ The number and extent of hemorrhagic events was determined by 3 independent treatment-blinded raters using a semi-quantitative method adapted from the ECASS classification as described elsewhere ${ }^{23,24}$ by giving 0 to 4 scores as follows: $0=$ absence of hemorrhage, 1-2 = increasing grades of hemorrhage infarction ( $\mathrm{HI} 1$ and $\mathrm{HI} 2$ ), and 3-4 $=$ homogeneous parenchymal hematomas of increasing grades (PH1 and $\mathrm{PH} 2)$; see Figure 3(d). The hemorrhage volume was also quantified with Image $\mathrm{J}$ following the same protocol as for the infarct volume.

\section{Functional tests}

Neurological deficits were evaluated in mice from Safety study 1 by a researcher blinded to the treatments with a 0 to 39 score based on a composite neurological scale $e^{25,26}$ at $24 \mathrm{~h}$ and $48 \mathrm{~h}$ after MCAo. The forelimb force was also assessed in the animals from Safety study 1 with the grip strength test as previously described. ${ }^{27}$ For details, please see the supplementary materials.

\section{Biochemical analysis}


Venous blood obtained from the right ventricle by cardiac puncture in EDTA tubes was centrifuged $\left(1500 \mathrm{~g}, 10 \mathrm{~min}\right.$ at $\left.4^{\circ} \mathrm{C}\right)$ to obtain plasma which was stored at $-80^{\circ} \mathrm{C}$ until analysis. Liver/pancreas/renal toxicity based on specific enzyme levels in plasma was assessed in the animals from Safety studies 1 and 2; for details see the supplementary materials.

\section{Statistical analysis}

Statistical analyses were performed using the GraphPad Prism 6 and SPSS softwares. The sample size of the Safety study 1 was determined in a pilot study ( $n=4-5 / g r o u p)$ by power analysis based on the infarct volume as primary outcome measure, using the software Ene 3.0 (GlaxoSmithKline SA), at $80 \%$ of statistical power and to a significance level of $5 \%$. Randomization of the experimental groups was generated using the standard $=$ RAND () function in Microsoft Excel. All values are expressed as mean $\pm S D$ or median (InterQuartile Range, IQR) according to the normal or nonnormal distribution of the represented variable, respectively. The normality of continuous variables was assessed using the Shapiro-Wilk test $(n<30)$ or Kolmogorov-Smirnov test $(n \geq 30)$. Normally distributed variables were analyzed using t-test or ANOVA (followed by Tukey's multiple comparisons post hoc test). The Mann-Whitney U-test or Kruskal Wallis test (followed by Dunn's multiple comparisons post hoc test) were used for non-normally distributed variables. Pearson's $x^{2}$ test or Fisher exact test were used for categorical variables. A total of 14 extreme values were excluded prior to data analyses using the outliers ROUT method $(Q=1 \%)$, corresponding to the biochemical parameters analysis $(n=11)$, the NC biodistribution in naïve mice $(n=2)$ and the safety NC administration in ischemic mice $(n=1)$. The significant level was set at $P<0.05$. 


\section{Results}

Intraarterial administration of nanomaterials shows a robust advantage for brain targeting

Our protocol for the endovascular administration of NC for brain targeting through the ICA in mice only transiently reduced the CBF at moderate levels for the first $10 \mathrm{~min}$ post-administration (34.8 $\pm 29.2 \%$ CBF reduction); see Figure 1 .

The different Cy7.5_NC batches used in this study presented a similar dose-dependent fluorescent signal when imaged ex vivo (Supplementary Figures S1(a) and S1(b)) and no associations between NC batch-fluorescence and treatment group were observed, discarding batch-dependent biased results for the FMI data (Supplementary Figures S1(c) and S1(d)).

Our results show a significant increase of the in vivo brain NC fluorescent signal (Total Radiant Efficiency, TRE) at all the acquisition time points after intraarterial administration compared to the intravenous route, both in the absence (F1) or presence (M1) of an external magnetic field $(P=$ $0.0062, P=0.0062$ and $P=0.0016$ for $\mathrm{F} 1$ at $30 \mathrm{~min}, 1 \mathrm{~h}$ and $3 \mathrm{~h}$ post-injection, respectively and $P=$ $0.0025, P=0.0025, P=0.0025$ for $\mathrm{M} 1$ at $30 \mathrm{~min}, 1 \mathrm{~h}$ and $3 \mathrm{~h}$ post-injection, respectively); see Figure 2(a) and Supplementary Figures S2(a) and S2(b). Figure 2(a) also shows the significant NC signal increase in the brain ex vivo at $3 \mathrm{~h}$ post-injection of animals receiving intraarterial infusions vs. intravenous $(P=0.0016$ for $\mathrm{F} 1$ and $P=0.004$ for M1). Specifically, the brain fluorescent signal at $3 \mathrm{~h}$ after intraarterial administration was increased $2.53 \pm 0.95$ times in vivo and $5.65 \pm 0.96$ ex vivo. Absolute fluorescence values were similar between administration routes in the abdominal region at all the in vivo acquisition time points and ex vivo in all organs (except the brain) at $3 \mathrm{~h}$ post-injection, and did not change in the presence of a magnetic field $(P>0.05$ for all measures, Supplementary 
Figure S2). However, when comparing the individual relative fluorescence in the abdominal region we found that it was significantly reduced in the intraarterial administration vs. the intravenous route $(P=0.0109, P=0.0062$ and $P=0.0031$ for $\mathrm{F} 1$ at $30 \mathrm{~min}, 1 \mathrm{~h}$ and $3 \mathrm{~h}$ post-injection, respectively and $P=0.0025, P=0.0025, P=0.0025$ for $\mathrm{M} 1$ at $30 \mathrm{~min}, 1 \mathrm{~h}$ and $3 \mathrm{~h}$ post-injection, respectively), and was also reduced in the liver ex vivo in the endovascular route with the magnet M1 $(P=$ 0.0012); see Supplementary Figure S3.

\section{Improved cortical NC targeting}

As shown in Figure 2(a), no differences were observed in the retention of the NC in the whole brain when implanting the magnet $\mathrm{M} 1$ for $30 \mathrm{~min}(P>0.05$ for $\mathrm{M} 1 \mathrm{vs}$. F1 for both administration routes). However, when dissecting the cortical and subcortical tissue we observed a significant increase $(P$ $=0.0382$; Figure 2(b)) in the brain fluorescence in the ipsilateral cortex when the magnetic field was applied by $\mathrm{M} 1$, in areas under the influence of the magnetic. Furthermore, a NC signal increase in the ipsilateral cortical and subcortical tissue was observed compared to the corresponding contralateral $(P<0.05$ for all measures; see Figure 2(b)).

\section{Acute endovascular administration of magnetized nanocarriers after cerebral ischemia is feasible and safe}

The safety study showed that the body weight loss and the mortality rate after MCAo were similar among NC and vehicle groups and within the expected values (Supplementary Figures S4(a) and S4(b)). Our results also showed no significant differences in functional outcome, infarct volume nor hemorrhagic transformations $(P>0.05$ for all measures) (Figure 3$)$.

Potential systemic toxicity of the endovascular-ICA administration of NC was studied at $48 \mathrm{~h}$ and 4 weeks after MCAo and no significant differences in the levels of alanine aminotransferase (ALT), 
lipase, creatinine, urea and sodium were observed among groups $(P>0.05$ for all measures). Only aspartate aminotransferase (AST) was increased at $48 \mathrm{~h}$ vs. the Naïve group $(P=0.031$ and $P=$ 0.031 for Vehicle and NC groups, respectively) and creatine kinase (CK), a muscular injury-related enzyme, was increased at $48 \mathrm{~h}$ in the NC treatment $v s$. the Naïve group $(P=0.027)$. Importantly, the increased levels of AST and CK in the vehicle and NC groups returned to normal levels at 4 weeks $(P>0.05$ for all comparisons), thus confirming the muscle injury-related increased levels of AST and CK acutely after the surgical intervention in the MCAo model. Finally, the levels of $\alpha$ amylase were increased at $48 \mathrm{~h}$ after the ischemia compared to the Naïve group $(P=0.0025$ for the NC group), returning to normal levels at 4 weeks $(P=0.0005$ vs. the NC group at $48 \mathrm{~h}$ postischemia). See Figure 4 and Supplementary Figure S4(c).

\section{Modelling magnetic field properties increases brain nanomaterial retention after ischemia}

We investigated the magnetic properties of the two FeNdB magnets designed as NC retention devices in the mouse brain, a permanent magnet (M1) and a focused magnet (M2) (Figures 5 and S5). Experimental measurements of the spatial magnetic field distributions at different distance from the surface of the M1 and M2 showed that the magnetic force decreases proportionally with the distance, displaying a higher magnetic force within the first $3 \mathrm{~mm}$ from the surface. However, M2 displays a 2.5 -fold increase of the maximum values of the magnetic field and 5.5 -fold increase of the magnetic force compared to $\mathrm{M} 1$, which is in agreement with the theoretical modelling of the magnetic field distribution of both magnet prototypes (Figure 5).

Next, we aimed to prove the in vivo effectiveness of M1 and M2 devices on magnetic brain targeting after acute endovascular NC administration at the calculated therapeutic dose after cerebral ischemia by FMI (Figure 6(a)). Our results show a significant $2.42 \pm 0.78$-fold increase of in vivo NC 
signal reaching the brain region at $3 \mathrm{~h}$ only by the $\mathrm{M} 2$ device compared with the $\mathrm{F} 2$ control $(P=$ $0.0209)$. This advantage was maintained at $48 \mathrm{~h}$ post-injection in vivo $(2.56 \pm 0.53$-fold increase, $P=$ $0.0001)$ and confirmed ex vivo $(2.16 \pm 0.8$-fold increase, $P=0.0398)$. On the other hand, the M1 device showed a $1.72 \pm 0.51$-fold increase in brain NC signal in vivo at $48 \mathrm{~h}$ post-injection compared to the F2 control, although not significant $(P=0.055)$. Importantly, M2 device showed $1.49 \pm 0.31$ times more brain NC signal at $48 \mathrm{~h}$ than $\mathrm{M} 1$ in vivo $(P=0.0182)$. In parallel, FMI values were similar in the abdominal region in vivo at $3 \mathrm{~h}$ and $48 \mathrm{~h}$ post-injection and ex vivo in all organs $(P>0.05$ for all measures, Supplementary Figure S6(b)).

From a translational perspective, we aimed to confirm the results by MRI, a clinically-relevant technique, to better define the spatial brain distribution of the NC after M2 implantation using the T2 relaxation properties of the SPION; see Figure 6(b). In accordance with the results observed by FMI, a significant increase in the particles count was observed in the ipsilateral cortex when the M2 device was implanted vs. the F2 control $(P=0.0125$; see Figure $6(b))$, thus confirming the success in magnetic retention of the NC in the brain cortex.

Finally, a specific Prussian blue staining was conducted to confirm the MRI findings, to identify the location of the nanomaterial in tissues. Representative images of brain, liver and spleen from animals receiving $\mathrm{NC}$ intraarterially in the presence of $\mathrm{M} 2$ are shown in Figure 7 and Supplementary Figure S7. Spleen slices were used as Prussian blue controls ${ }^{28}$ and liver slices were also stained to confirm the presence of the NC in tissues as NC typically accumulate in the liver (Supplementary Figure S7). Brain slices showed extensive positive staining in the ipsilateral brain hemisphere, predominantly in microvessel-like structures, while the presence of ferric iron in the contralateral hemisphere was negligible, matching with the FMI and MRI results (see Figure 7). Additionally, the 
predominant distribution of NC in ipsilateral brain microvessels was confirmed by co-localization of the Cy7.5 fluorescence with lectin-FITC stained microvessels (Figure 7).

\section{Administration-related cautions}

Adverse events have been previously described after intraarterial cell administration such as microocclusions and increased mortality rate. ${ }^{12,29,30}$ In this regard, it should be highlighted that the average size of the nanomaterial used in the present study was $273.21 \pm 34.7 \mathrm{~nm}$, within an acceptable range to avoid embolization complications. Indeed, no differences were observed in the mortality rate between ischemic mice receiving NC or vehicle intraarterially (Safety study 1: $21.1 \%$ and $27.3 \%$, respectively $(P=0.727)$; see Supplementary Figure S4(b)). Importantly, we observed a trend $(P=0.091)$ between the total iron load dose and the mortality rate among ischemic C57 mice receiving NC intraarterially, which alerts us on potential harms of the use of certain nanomaterials.

\section{Discussion}

In this study, we tested the use of biocompatible nanomaterials to improve brain targeting after stroke taking advantage of the endovascular route to reach the brain vasculature and the use of functionalized nanocarriers for imaging and magnetic targeting. Our data prove the safety and feasibility of acute brain endovascular infusion of multimodal biocompatible PLGA-NC in a mouse model of cerebral ischemia to significantly improve brain delivery, paving the way for future nanomaterial-based treatments in the context of EVT.

A crucial aspect to consider while investigating a new therapeutic approach is the ideal administration route in terms of safety and efficient delivery while considering the particularities of a given pathological condition. As mentioned above, endovascular treatments have the advantage of 
a more selective targeting while minimizing the side effects of systemic drug delivery in filtering organs. ${ }^{11,12}$ In consistency with previous studies, ${ }^{20,31,32}$ we have observed that intravenously administered NC predominantly accumulate in the liver, lungs and spleen, while their retention in the brain is almost negligible. In contrast, our data confirm the significant advantage for strokeaffected brain tissue targeting following the intraarterial-ICA administration route, ${ }^{33,34}$ as seen both by MRI and FMI when delivering small nanocarriers (below $300 \mathrm{~nm}$ in our study). The preferential ipsilateral and negligible contralateral hemisphere NC accumulation, seen both in healthy and ischemic mice in our study, has been already described by Lesniak and colleagues observing a preferential ipsilateral accumulation of Bevacizumab after intraarterial administration of the free antibody, which was further enhanced following the blood-brain barrier (BBB) opening with mannitol and preserving the preference for the ipsilateral hemisphere of the injection. ${ }^{35}$ Further investigations are required for a deeper understanding of the underlying mechanisms for this specific ipsilateral uptake and to fully elucidate the effects of this approach on the integrity of the BBB. However, we did not observe major hemorrhagic events after intraarterial vehicle nor NC administration following MCAo, evidencing the safety in the context of cerebral ischemia. Similarly, the SAVER-I Phase I trial has also demonstrated the feasibility and safety of the intraarterial administration of free verapamil after standard thrombectomy with no evidence of hemorrhagic transformations. ${ }^{36}$ Brain delivery of therapeutic agents in minimally invasive approaches has been a major challenge which could be faced with new nanomedicine tools. Due to the inherent nanocarriers accumulation in filtering organs, many strategies have been focused on adding affinity moieties to increase the specific accumulation in target organs but have been hindered by numerous challenges. A recent study has nicely reported a substantial increase of nanocarriers accumulation in the brain by using 
red blood cells as a vascular carrier for NC and administration through the ICA, confirming the enormous advantage of this endovascular delivery route in downstream organs, which can be further improved with nanomedicine-based strategies for brain targeting. ${ }^{37}$ In this light, seizing the advantages of polymeric nanoparticles to administer therapeutic agents with a sustained release, increased half-life in vivo and reduced systemic toxic effects, ${ }^{38}$ we have used a PLGA-based nanocarrier with biocompatible properties which has been functionalized with SPION and Cy7.5 for a magnetically guided retention and in vivo tracking by MRI and FMI as previously described by our group. ${ }^{20}$ In our opinion, the use in the present study of biocompatible approved biomaterials for the medical use as PLGA and SPIONs with improved magnetic targeting is a potential advantage for the clinical translation of nanomedical products, however other non-magnetized PLGA formulations or alternative biogenic / synthetized biomaterials could be used to achieve the successful brain delivery using the endovascular route after stroke.

To further improve the guided transport of the used nanocarriers, NC were functionalized with SPION to allow their retention by a magnetic field device to specific brain areas which was proved by our FeNdB devices in cortical areas under magnetic influence, with particular success with the focused magnet design (M2) which showed better performance related to higher magnetic field and magnetic field gradient in deeper positions, as previously described. ${ }^{39,40}$ This is the first time that a focused magnet designed for mouse brains is used to target the cortical delivery of nanocarriers after stroke in endovascular-ICA administrations, although other authors have also used an external magnet to enhance the delivery of magnetized cells to specific organs, including the brain after intravenous stem cell administration and BBB opening in a rat model of traumatic brain injury. ${ }^{41}$ 
Our results first failed in showing an advantage in magnetic targeting using permanent magnets (M1) and short implantation times (30 min) in whole hemispheres. Nonetheless, finest experiments further proved the increased NC retention in the cortex after the advantageous intraarterial-ICA administration, by focusing on the specific area of magnetic field influence (several $\mathrm{mm}$, corresponding to the cortical brain areas) with FMI and better spatial resolution in MRI using the improved focused magnet (M2). It is worth noting that we have also been able to detect by different in vivo and ex vivo imaging techniques the presence of the NC in the brain up to $48 \mathrm{~h}$ after the endovascular infusion, suggesting that a substantial proportion of the administered NC remains available in the target organ for a long time period, easing the sought-after sustained release of encapsulated therapeutic agents. Interestingly, even though a substantial amount of the NC still remains in filtering organs such as the lungs, liver and spleen, our study also proves that the endovascular administration diminished the accumulation of NC in the liver, a known off-target accumulation of nanomaterials in filtering organs. Importantly, this unavoidable accumulation of the NC observed in our study did not alter known biomarkers of liver/pancreas/renal damage at shortnor at long-term after intraarterial infusion compared to healthy mice, and only alterations related to the surgical intervention were found transiently altered, supporting the safety of the NC treatment in terms of systemic toxicity.

As previously mentioned, the pre-clinical use of the intraarterial administration route has raised safety concerns in previous investigations since thromboembolisms have been described as a common related complication, mainly associated with the infusion rate, dose or cell size. ${ }^{12,29,42}$ To prevent this major complication, NC with an average diameter below $300 \mathrm{~nm}$ were freshly prepared in a saline suspension, vortexed and sonicated before the endovascular infusion to avoid the NC 
aggregation. The CBF, which was monitored during a controlled infusion, was only transiently and moderately reduced, as similarly reported in previous studies using mesenchymal stem cells by intraarterial administration, ${ }^{29,42}$ but the intraarterial-ICA infusion of NC was not associated to mortality, a worsening of the ischemic damage nor a higher risk of hemorrhages, suggesting a low risk of thromboembolisms. Nonetheless, from a translational perspective, future pre-clinical studies should refine these endovascular delivery approaches and study the observed cerebral blood flow changes with advanced imaging techniques. On the other hand, the maintenance of iron homeostasis in the context of cerebral ischemia is of great importance, as iron catalyzes the formation of free radicals and hence, can cause oxidative stress and worsen the ischemic damage. ${ }^{43}$ Importantly, we did not observe an increase in the infarct volume nor hemorrhages following the acute endovascular-ICA NC administration (containing SPION) after stroke compared to the vehicle infusion, suggesting the safety in the tested conditions. Nevertheless, we observed a trend in higher total iron load dose and increased mortality only in ischemic mice but not in naïve, indicating a potential iron load-related risk in the context of acute ischemic cerebral damage. Hence, the iron load should be strictly adjusted when using magnetized nanocarriers as drug delivery systems in the context of cerebral ischemia. Although we have shown some data regarding long term safety at 2 weeks, additional studies with long-term monitoring of the administered NC in terms of safety and biodistribution are needed. Furthermore, our histological findings suggest an endothelial uptake of the nanocarriers, but other cellular or extracellular localizations within the brain parenchyma upon endovascular administration and magnetic brain targeting is yet to be investigated. In this light, this approach could be further combined with promising strategies aiming at the BBB crossing of therapeutic agents, such as the selective BBB temporary opening with 
nanoagonists such as adenosine $2 \mathrm{~A}$ receptor agonist-labelled PAMAM dendrimers ${ }^{44}$ or the functionalization of nanoparticles with selective antibodies for a BBB receptor-mediated transcytosis. ${ }^{45}$ Finally, the present investigation provides evidence supporting the potential use and advantages of the endovascular delivery of PLGA nanocarriers which have already proven by our group and others the versatility for the encapsulation or adsorption of a large variety of proteins, ranging from 8 to $66.5 \mathrm{kDa}$ (e.g. VEGF, SDF, BDNF or albumin), showing successful encapsulation efficiencies, and posterior in vitro release with preserved functionality of the released factors. ${ }^{46-48}$ This supports the encapsulation of therapeutic molecules for tissue repair, for example, however any potential restriction on the molecular weight of the cargo proteins should be further studied for specific neuroprotective or neurorestorative agents, always in balance with suitable nanoformulation sizes for a safe endovascular delivery and proving its advantage in front of free drug deliveries. The present study proves the advantage and safety of intraarterially delivered magnetized nanocarriers for specific brain targeting in the context of cerebral ischemia. Nonetheless, there are several limitations that should be considered for future studies. The biodistribution and safety of the proposed nanocarriers should be readdressed for different encapsulated molecules or the addition/removal of imaging or affinity moieties. Furthermore, the iron load-derived toxicity, as well as the clearance mechanism and kinetics of this type of magnetic nanocarrier is yet to be fully elucidated. Finally, from a translational perspective, studies in large animals with gyrencephalic brains and larger vessels/capillaries or in humanized prototypes should be used to demonstrate the feasibility of the proposed therapy. 


\section{Acknowledgments}

We are grateful for the technical assistance received from the Pre-clinical imaging Platform at Vall d'Hebron Institut de Recerca, the Servei RMN at Universitat Autònoma de Barcelona, and the Unitat de Microscopia Òptica Avançada, Facultat de Medicina at the Universitat de Barcelona.

This work has been supported under the Euronanomed MAGGBRIS collaborative project by grants from the Spanish Ministry of Science and Innovation (PCIN-2017-090 grant), the Instituto de Salud Carlos III (AC17/00004 with FEDER funds), the Slovak Research and Development Agency under the Contract No.APVV-19-0324 and the Italian Ministry of Health (Ricerca Corrente year 2017 funds); by the Expression of Interest (Eol) for Collaborative Projects on Regenerative Medicine 2019 P-CMR[C]); programs 2017-SGR-1427 and 2017-SGR-765 from the Generalitat de Catalunya; RETICS-INVICTUS PLUS from ISCIII (RD16/0019/0021 with FEDER funds); the 'Severo Ochoa' Program for Centers of Excellence in R\&D (SEV-2015-0496) and the RYC-201722412 and PID2019-107989RB-I00. A.G has been supported by fellowships from ISCIII (FI17/00073 and MV18/00006), A.R by a visiting-scientist fellowship from ISCIII (BA17/00052) and Y. Z has been supported by the China Scholarship Council (CSC).

\section{Author Contributions}

A.R.N. and A.R.S. contributed to the study conception; A.G., A.R.N., A.R.S., P.K., R.M.M. and M.P. contributed to the study design; A.G., M.G. and M.A., A.M., M.T., J.K, O.S. and Y.Z conducted the experimental studies; A.G., M.T. and J.K. analyzed the data, A.G. and A.R.N. wrote the paper and all authors contributed to the draft revisions. 
Declaration of conflict of interests: The authors declare no conflicts of interest.

\author{
Supplementary material \\ Supplementary material for this paper can be found at \\ http://jcbfm.sagepub.com/content/by/supplemental-data
}

\title{
Data availability
}

Data related to this manuscript will be available upon reasonable request to the corresponding author.

\section{References}

1. Maingard J, Foo M, Chandra R V., et al. Endovascular Treatment of Acute Ischemic Stroke. Curr Treat Options Cardiovasc Med 2019; 21: 225-558.

2. Haussen DiC, Lima A, Nogueira RG. The Trevo XP 320 mm retriever ('Baby Trevo') for the treatment of distal intracranial occlusions. J Neurointerv Surg 2016; 8(3): 295-299.

3. Schwaiger BJ, Kober F, Gersing AS, et al. The pREset Stent Retriever for Endovascular Treatment of Stroke Caused by MCA Occlusion: Safety and Clinical Outcome. Clin Neuroradiol 2016; 26(1): 47-55.

4. Kaneko N, Komuro $\mathrm{Y}$, Yokota $\mathrm{H}$, et al. Stent retrievers with segmented design improve the efficacy of thrombectomy in tortuous vessels. J Neurointerv Surg 2019; 11(2): 119-122.

5. Fennell VS, Setlur Nagesh SV, Meess KM, et al. What to do about fibrin rich "tough clots"? 
Comparing the Solitaire stent retriever with a novel geometric clot extractor in an in vitro stroke model. J Neurointerv Surg 2018; 10(9): 907-910.

6. Gruber P, Zeller S, Garcia-Esperon C, et al. Embolus Retriever with Interlinked Cages versus other stent retrievers in acute ischemic stroke: An observational comparative study. $\mathrm{J}$ Neurointerv Surg 2018; 10(12): e31.

7. Powers WJ, Rabinstein AA, Ackerson $T$, et al. Guidelines for the early management of patients with acute ischemic stroke: 2019 update to the 2018 guidelines for the early management of acute ischemic stroke a guideline for healthcare professionals from the American Heart Association/American Stroke A. Stroke 2019; 50(12): e344-e418.

8. Nogueira RG, Jadhav AP, Haussen DC, et al. Thrombectomy 6 to 24 hours after stroke with a mismatch between deficit and infarct. N Engl J Med 2018; 378(1): 11-21.

9. Albers GW, Marks MP, Kemp S, et al. Thrombectomy for stroke at 6 to 16 hours with selection by perfusion imaging. N Engl J Med 2018; 378(8): 708-718.

10. Gittler M, Davis AM. Guidelines for adult stroke rehabilitation and recovery. JAMA 2018; 319(8): 820-821.

11. Griauzde J, Ravindra VM, Chaudhary N, et al. Neuroprotection for ischemic stroke in the endovascular era: A brief report on the future of intra-arterial therapy. J Clin Neurosci 2019; 69: 289-291.

12. Guzman R, Janowski M, Walczak P. Intra-arterial delivery of cell therapies for stroke. Stroke 2018; 49(5): 1075-1082.

13. Bhatia V, Gupta V, Khurana D, et al. Randomized assessment of the safety and efficacy of intra-arterial infusion of autologous stem cells in subacute ischemic stroke. AJNR Am J 
Neuroradiol 2018; 39(5): 899-904.

14. Fraser JF, Maniskas M, Trout A, et al. Intra-arterial verapamil post-thrombectomy is feasible, safe, and neuroprotective in stroke. J Cereb Blood Flow Metab 2017; 37(11): 3531-3543.

15. Wu C, Zhao W, An H, et al. Safety, feasibility, and potential efficacy of intraarterial selective cooling infusion for stroke patients treated with mechanical thrombectomy. J Cereb Blood Flow Metab 2018; 38(12): 2251-2260.

16. Danhier F, Ansorena E, Silva JM, et al. PLGA-based nanoparticles: An overview of biomedical applications. J Control Release 2012; 161(2): 505-522.

17. Wang Y, Qu W CS. FDA's regulatory science program for generic PLA/PLGA-based drug products. Am Pharm Rev 2017; 19: 5-9.

18. du Sert NP, Hurst V, Ahluwalia A, et al. The ARRIVE guidelines 2.0: Updated guidelines for reporting animal research. J Cereb Blood Flow Metab 2020; 40(9): 1769-1777.

19. Clark WM, Lessov NS, Dixon MP, et al. Monofilament intraluminal middle cerebral artery occlusion in the mouse. Neurol Res 1997; 19(6): 641-648.

20. Zhang Y, García-Gabilondo M, Grayston A, et al. PLGA protein nanocarriers with tailormade fluorescence/MRI/PET imaging modalities. Nanoscale 2020; 12(8): 4988-5002.

21. Hundt W, Petsch R, Helmberger T, et al. Signal changes in liver and spleen after Endorem administration in patients with and without liver cirrhosis. Eur Radiol 2000; 10(3): 409-416.

22. Morancho A, García-Bonilla L, Barceló V, et al. A new method for focal transient cerebral ischaemia by distal compression of the middle cerebral artery. Neuropathol Appl Neurobiol 2012; 38(6): 617-627.

23. García-Yébenes I, Sobrado M, Zarruk JG, et al. A mouse model of hemorrhagic 
transformation by delayed tissue plasminogen activator administration after in situ thromboembolic stroke. Stroke 2011; 42(1): 196-203.

24. Campos M, García-Bonilla L, Hernández-Guillamon M, et al. Combining statins with tissue plasminogen activator treatment after experimental and human stroke: A safety study on hemorrhagic transformation. CNS Neurosci Ther 2013; 19(11): 863-870.

25. Orsini F, Villa P, Parrella S, et al. Targeting mannose-binding lectin confers long-lasting protection with a surprisingly wide therapeutic window in cerebral ischemia. Circulation 2012; 126(12): 1484-1494.

26. De Simoni MG, Storini C, Barba M, et al. Neuroprotection by complement (C1) inhibitor in mouse transient brain ischemia. J Cereb Blood Flow Metab 2003; 23(2): 232-239.

27. Rosell A, Agin V, Rahman M, et al. Distal Occlusion of the Middle Cerebral Artery in Mice: Are We Ready to Assess Long-Term Functional Outcome? Transl Stroke Res 2013; 4(3): 297-307.

28. Sukhbaatar N, Weichhart T. Iron regulation: Macrophages in control. Pharmaceuticals 2018; 11(4): 137.

29. Cui LL, Kerkelä E, Bakreen A, et al. The cerebral embolism evoked by intra-arterial delivery of allogeneic bone marrow mesenchymal stem cells in rats is related to cell dose and infusion velocity. Stem Cell Res Ther 2015; 6(1): 11.

30. Argibay B, Trekker J, Himmelreich $\mathrm{U}$, et al. Intraarterial route increases the risk of cerebral lesions after mesenchymal cell administration in animal model of ischemia. Sci Rep 2017; 7: 40758.

31. Cruz LJ, Stammes MA, Que I, et al. Effect of PLGA NP size on efficiency to target traumatic 
32. Wiley DT, Webster P, Gale A, et al. Transcytosis and brain uptake of transferrin-containing nanoparticles by tuning avidity to transferrin receptor. Proc Natl Acad Sci USA 2013; 110: 8662-8667. brain injury. J Control Release 2016; 223: 31-41.

33. Pendharkar A V., Chua JY, Andres RH, et al. Biodistribution of neural stem cells after intravascular therapy for hypoxic-ischemia. Stroke 2010; 41(9): 2064-2070.

34. Li L, Jiang Q, Ding G, et al. Effects of administration route on migration and distribution of neural progenitor cells transplanted into rats with focal cerebral ischemia, an MRI study. J Cereb Blood Flow Metab 2010; 30(3): 653-662.

35. Lesniak WG, Chu C, Jablonska A, et al. PET imaging of intra-arterial $89 \mathrm{Zr}$ bevacizumab in mice with and without osmotic opening of the blood-brain barrier: distinct advantage of intraarterial delivery. J Nucl Med 2018; 60(5): 617-622.

36. Fraser JF, Maniskas M, Trout A, et al. Intra-arterial verapamil post-thrombectomy is feasible, safe, and neuroprotective in stroke. J Cereb Blood Flow Metab 2017; 37(11): 3531-3543.

37. Brenner JS, Pan DC, Myerson JW, et al. Red blood cell-hitchhiking boosts delivery of nanocarriers to chosen organs by orders of magnitude. Nat Commun 2018; 9(1): 2684 .

38. Ding D, Zhu Q. Recent advances of PLGA micro/nanoparticles for the delivery of biomacromolecular therapeutics. Mater Sci Eng C Mater Biol Appl 2018; 92: 1041-1060.

39. Kopcansky P, Timko M, Hnatic M, et al. Numerical modeling of magnetic drug targeting. Phys Part Nucl Lett 2011; 8: 502-505.

40. Kopćanský P, Bánó M, Repašan M, Potoċová I, Timko M, Hrnċiar V, Demjan S. Magnetic targeted drug delivery using focused magnet. Magnetohydrodynamics 2004; 40: 369-378. 
41. Shen W Bin, Anastasiadis P, Nguyen B, et al. Magnetic enhancement of stem cell-targeted delivery into the brain following MR-guided focused ultrasound for opening the blood-brain barrier. Cell Transplant 2017; 26: 1235-1246.

42. Walczak $\mathrm{P}$, Zhang J, Gilad AA, et al. Dual-modality monitoring of targeted intraarterial delivery of mesenchymal stem cells after transient ischemia. Stroke 2008; 39: 1569-1574.

43. Minhas G, Modgil S, Anand A. Role of iron in ischemia-induced neurodegeneration: mechanisms and insights. Metab Brain Dis 2014; 29: 583-591.

44. Gao X, Wang YC, Liu Y, Yue Q, Liu Z, Ke M, Zhao S, Li C. Nanoagonist-mediated endothelial tight junction opening: A strategy for safely increasing brain drug delivery in mice. J Cereb Blood Flow Metab 2017; 37(4): 1410-1424.

45. Yemisci M, Caban S, Gursoy-Ozdemir Y, Lule S, Novoa-Carballal R, Riguera R, FernandezMegia E, Andrieux K, Couvreur P, Capan Y, Dalkara T. Systemically administered braintargeted nanoparticles transport peptides across the blood-brain barrier and provide neuroprotection. J Cereb Blood Flow Metab 2015; 35(3): 469-75.

46. Zhang Y, García-Gabilondo M, Rosell A, et al. MRI/Photoluminescence Dual-Modal Imaging Magnetic PLGA Nanocapsules for Theranostics. Pharmaceutics 2019; 12(1): 16.

47. Carenza E, Jordan O, Martínez-San Segundo P, et al. Encapsulation of VEGF165 into magnetic PLGA nanocapsules for potential local delivery and bioactivity in human brain endothelial cells. J Mater Chem B 2015; 3(12): 2538-2544.

48. Pakulska MM, Donaghue IE, Obermeyer JM, et al. Encapsulation-free controlled release: Electrostatic adsorption eliminates the need for protein encapsulation in PLGA nanoparticles. Sci Adv 2016; 2(5): e1600519. 


\section{Figure legends}

Figure 1. Endovascular-ICA administration of functionalized NC. (a) Scheme of the subcutaneous magnet implantation and the surgical procedure for intraarterial NC administration. (b) CBF during and after intraarterial administration of $0.8 \mathrm{mg}$ of NC in $150 \mu \mathrm{L}$ of saline $(n=6)$. Data shown as mean $\pm S D$. (c) Representative SEM image of NC. Inset showing a single NC TEM image, with SPIONs visible as black spots uniformly distributed in the PLGA. Abbreviations: PA: pterygopalatine artery.

Figure 2. Biodistribution after NC administration showing the advantage of intraarterial-ICA route and magnetic targeting in naïve mice. (a) Scheme of the Biodistribution study 1 , representative in vivo and ex vivo FMl images at $3 \mathrm{~h}$ after intravenous/intraarterial NC injection and graphs showing the corresponding quantification on brain regions of interest (ROIs). Data shown as median (IQR). (b) Scheme of the Biodistribution study 2, representative images ex vivo FMI of the ipsilateral/contralateral cortical and subcortical brain regions at $3 \mathrm{~h}$ after intraarterial NC injection and graphs showing the corresponding quantification. Data shown as median (IQR). ${ }^{*} \mathrm{P}<0.05$, ${ }^{* *} \mathrm{P}<0.01,{ }^{* \star *} \mathrm{P}<0.001$. Abbreviations: i.v.: intravenous, i.a.: intraarterial, Ctrl: control, ILC: ipsilateral cortex, ILSC: ipsilateral subcortex, CLC: contralateral cortex, CLSC: contralateral subcortex.

Figure 3. Safety of acute intraarterial-ICA NC administration after MCAo and magnetic targeting. (a) Scheme of the experimental design of Safety study 1. (b) Graphs showing infarct and hemorrhage volumes. (c) Graphs showing neuroscore and grip strength measurements. (d) 
Hemorrhages evaluation by visual classification according to ECASS criteria in TTC-stained brains represented as the percentage of animals classified in each category. Data represented in Infarct volume and Grip strength show the mean \pm SD, and data represented in Hemorrhage volume and Neuroscore graphs show the median (IQR). n.s.: non-significant.

Figure 4. Systemic toxicity study at $48 \mathrm{~h}$ and 4 weeks after MCAo and intraarterial-ICA NC administration. (a) Scheme of the experimental design of Safety studies 1 and 2. (b) Plasma levels of AST, ALT and CK All data represented in graphs show the median (IQR), except the graphs showing AST levels at $48 \mathrm{~h}$ and $\mathrm{CK}$ levels at 4 weeks, the data showing mean $\pm S D$. ${ }^{*} P<0.05$, n.s.: non-significant. Abbreviations: V: vehicle.

Figure 5. Characterization of the magnetic field and magnetic force of the magnet devices.

(a) Spatial distribution by computational modelling of the magnetic field and spatial distribution of the magnetic force for M1 (left) and M2 (right) at $3 \mathrm{~mm}$ above the magnet surface. (b) Graph (left) showing the magnetic field ( $B$; dotted lines) and magnetic force $\left(B^{*} d B\right.$; straight lines) of $M 1$ (black) and M2 (red) at $3 \mathrm{~mm}$ above the magnet surface, measured by 3D Hall probe. (c) Image (above) of M1 and M2 devices and illustrative image (below) of the dust effectivity test of magnetic targeting of ferrimagnetic magnetite microparticles for M1 and M2.

Figure 6. Biodistribution after MCAO and intraarterial-ICA NC administration showing the improved NC brain targeting with different magnetic devices and superior performance of the focused magnet design (M2). (a) Scheme of the experimental Biodistribution study 3, 
representative in vivo FMI images and ex vivo after intraarterial-ICA NC administration and graphs showing the corresponding quantification on brain ROIs. (b) Scheme of the Biodistribution study 4 , representative brain $\mathrm{T} 2{ }^{*} \mathrm{WI} 48 \mathrm{~h}$ after intraarterial-ICA NC administrationand graphs showing the corresponding particle analysis of the hypointense signals attributed to SPIONs in ipsilateral/contralateral cortical and subcortical regions. All data are shown as mean $\pm S D$, except the graph showing the particles count in the subcortical regions, where data is represented as median (IQR). \#P<0.01, ${ }^{*} \mathrm{P}<0.05,{ }^{* *} \mathrm{P}<0.01,{ }^{* * *} \mathrm{P}<0.001$.

Figure 7. Verification of NC brain distribution by Prussian blue ferric iron staining (SPION) and fluorescence (Cy7.5). Staining on brain shows a major and extensive signal in the ipsilateral hemisphere; note that most positive stain corresponds to microvessel-like structures in salineperfused brains. Scale bar: $20 \mu \mathrm{m}$. 


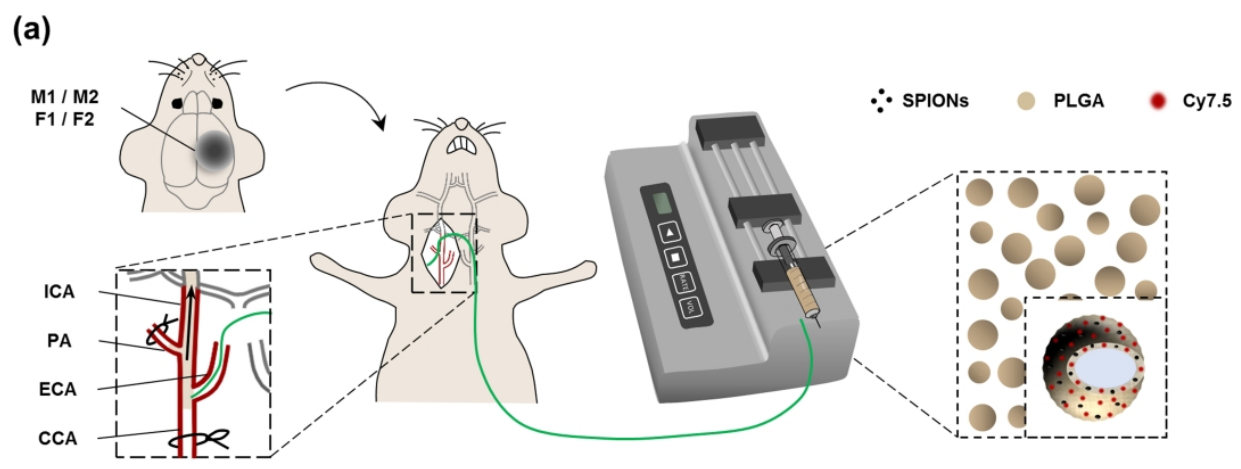

(b)

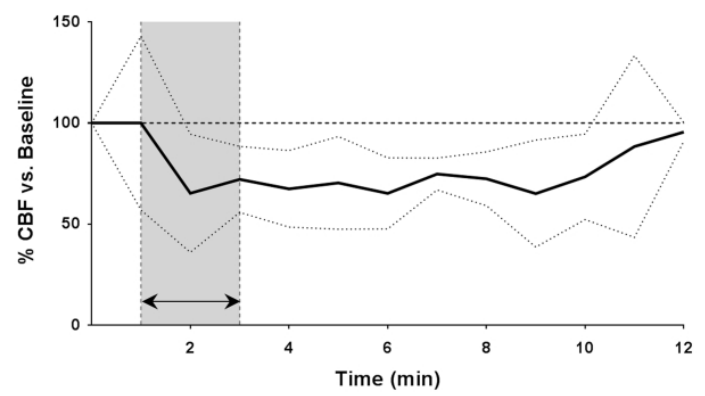

(c)

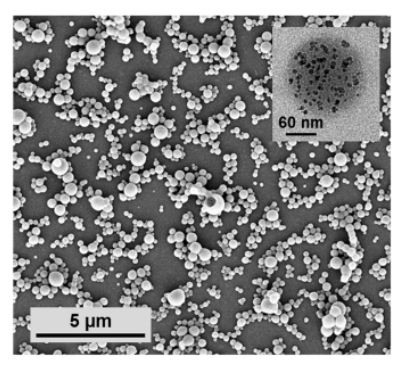

Figure 1. Endovascular-ICA administration of functionalized NC.

$172 \times 129 \mathrm{~mm}(500 \times 500 \mathrm{DPI})$ 

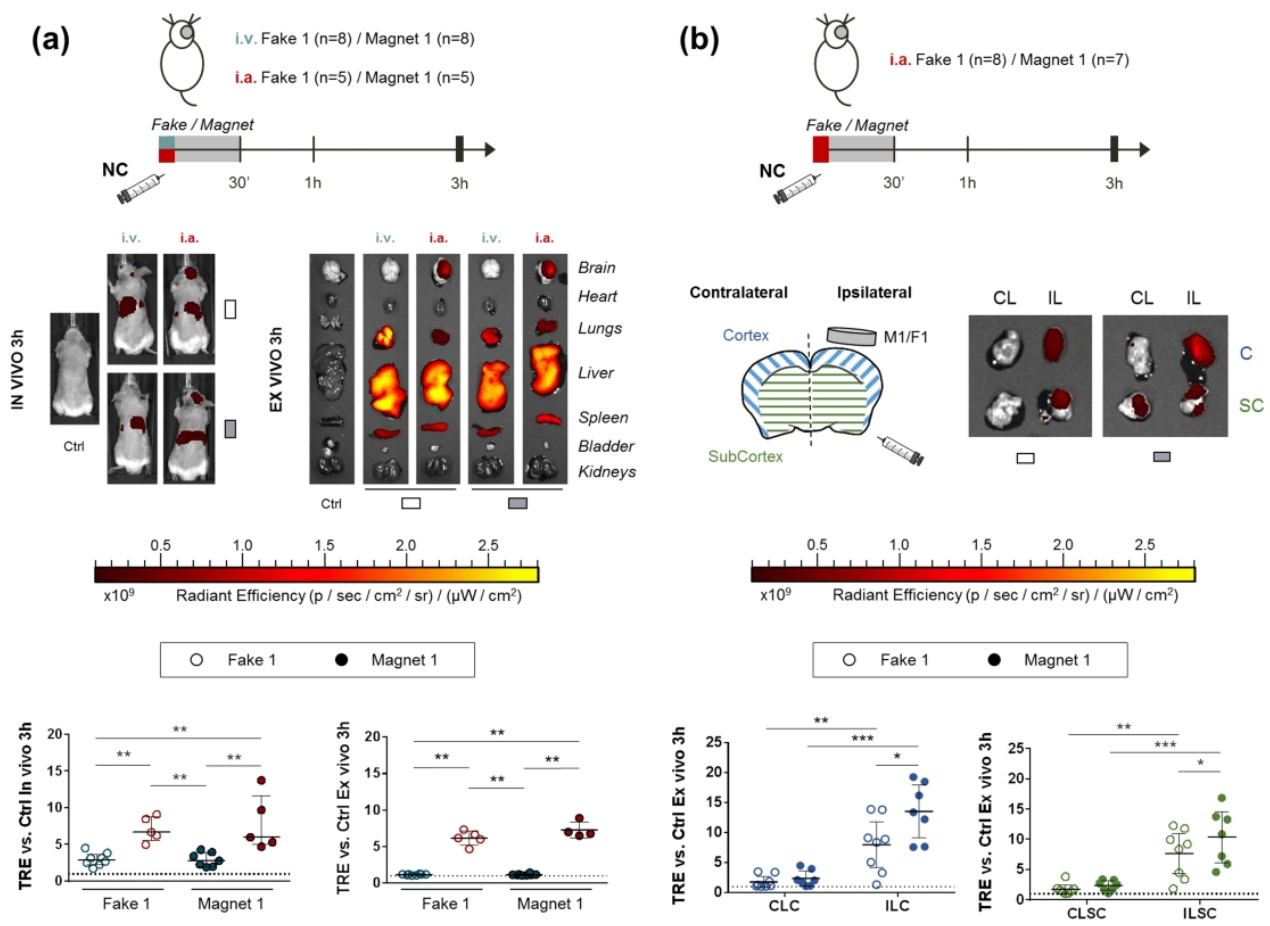

Figure 2. Biodistribution after NC administration showing the advantage of intraarterial-ICA route and magnetic targeting in naïve mice.

$172 \times 127 \mathrm{~mm}(500 \times 500 \mathrm{DPI})$ 
(a)
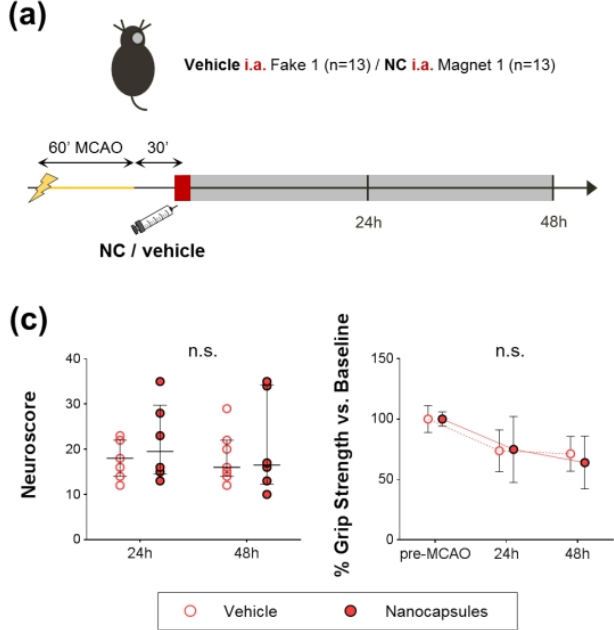

(b)

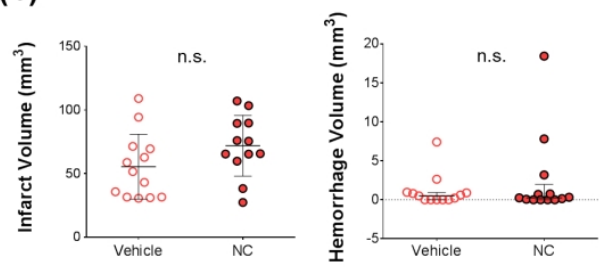

(d)

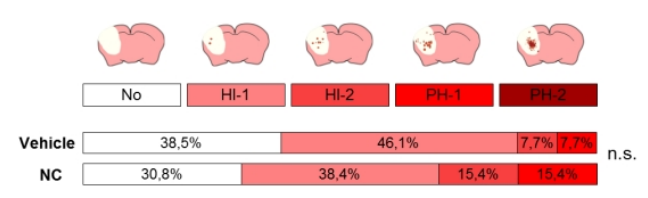

Figure 3. Safety of acute intraarterial-ICA NC administration after MCAo and magnetic targeting.

$172 \times 90 \mathrm{~mm}(300 \times 300 \mathrm{DPI})$ 
(a)

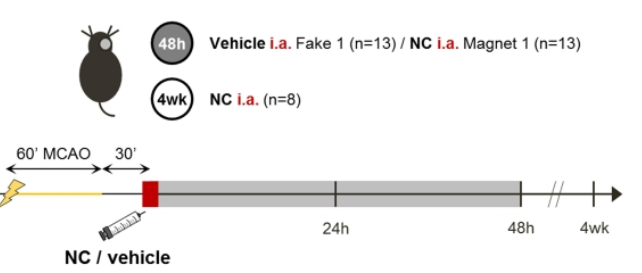

(b)

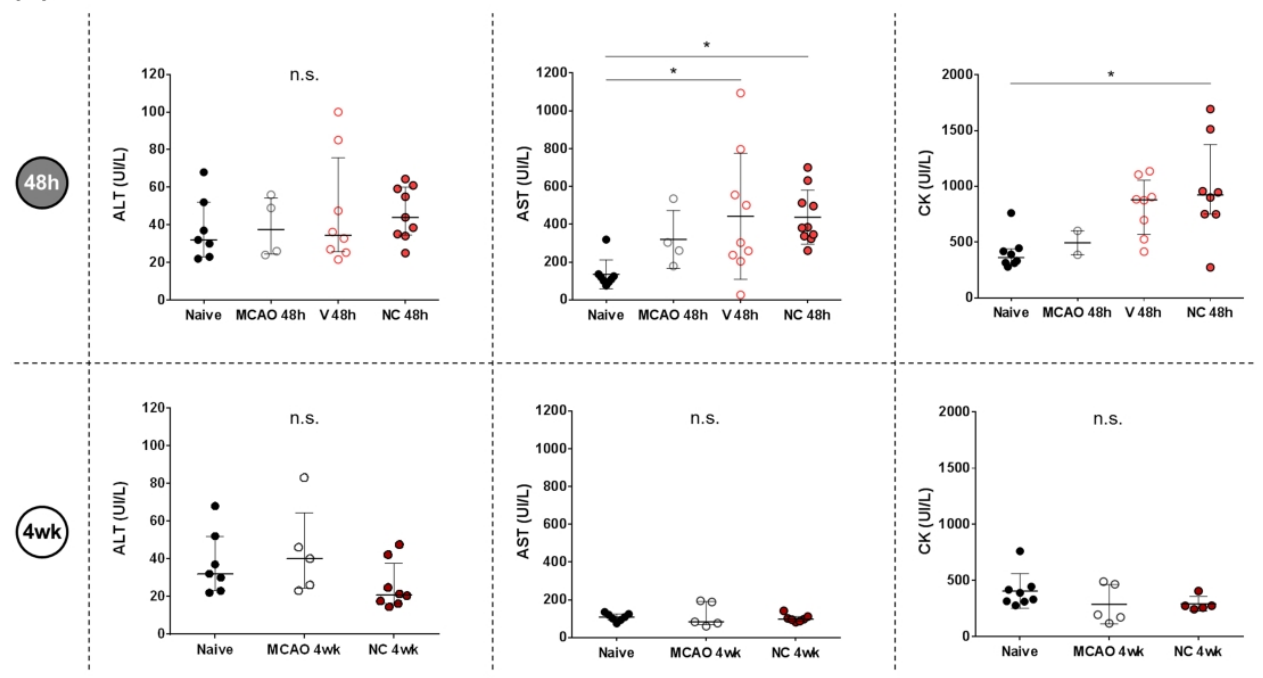

Figure 4. Systemic toxicity study at $48 \mathrm{~h}$ and 4 weeks after MCAo and intraarterial-ICA NC administration. $172 \times 139 \mathrm{~mm}(800 \times 800 \mathrm{DPI})$ 

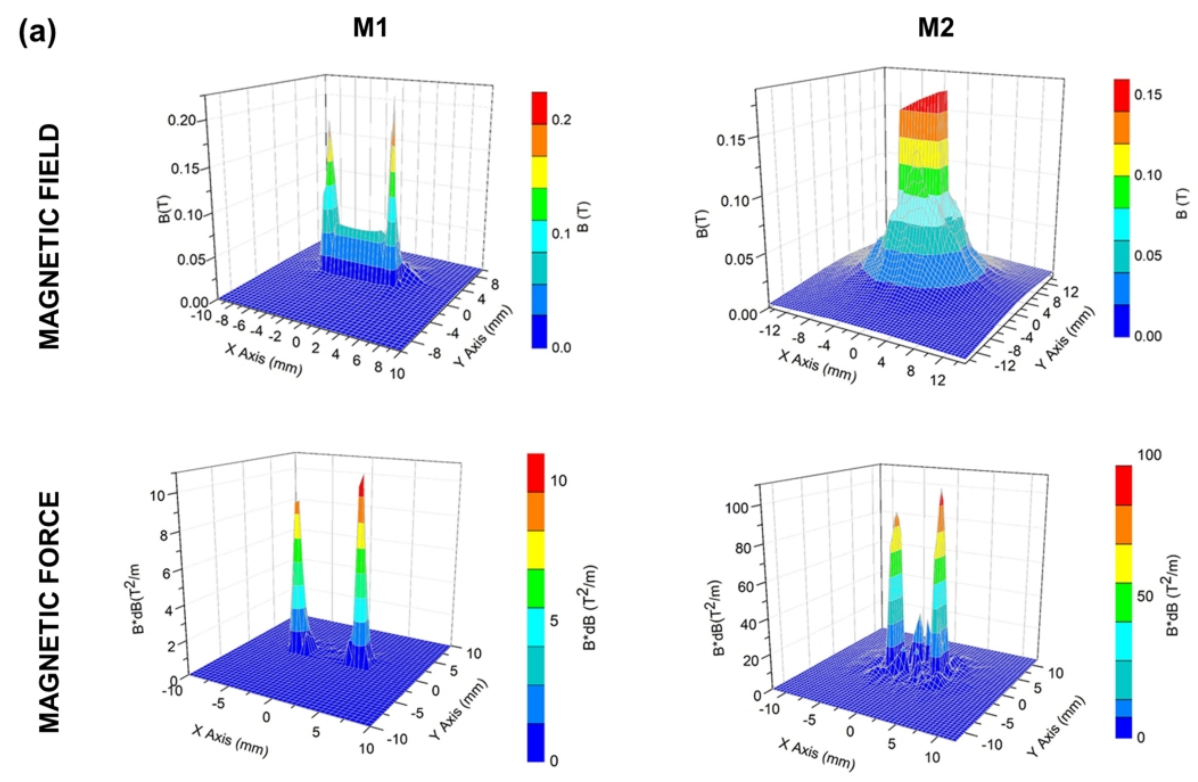

(b)

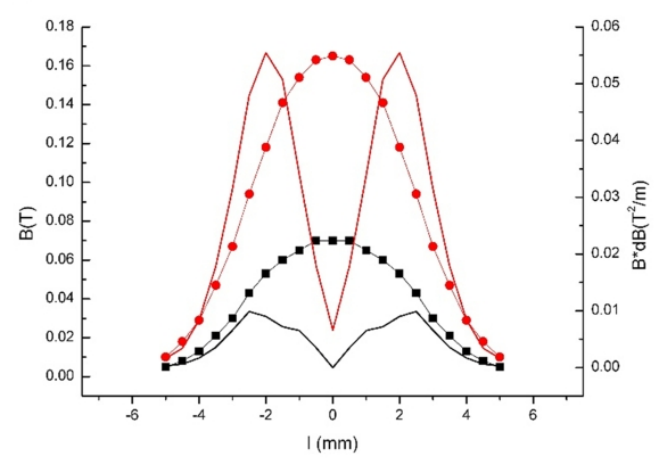

(c)

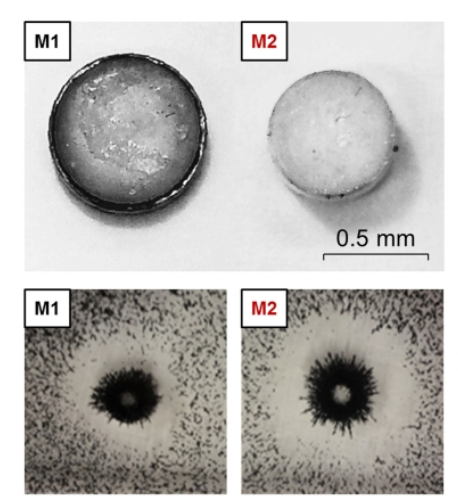

Figure 5. Characterization of the magnetic field and magnetic force of the magnet devices.

$172 \times 180 \mathrm{~mm}(600 \times 600 \mathrm{DPI})$ 
(a)
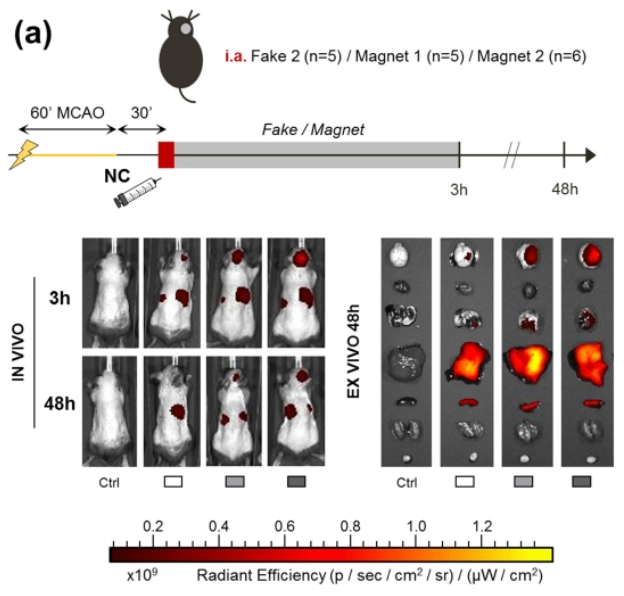

\begin{tabular}{|llllll|}
\hline$\circ$ & Fake 2 & $\circ$ & Magnet 1 & $\bullet$ & Magnet 2 \\
\hline
\end{tabular}
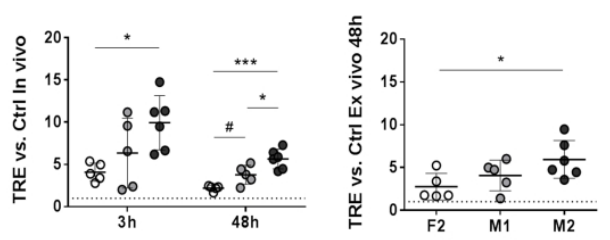

(b)
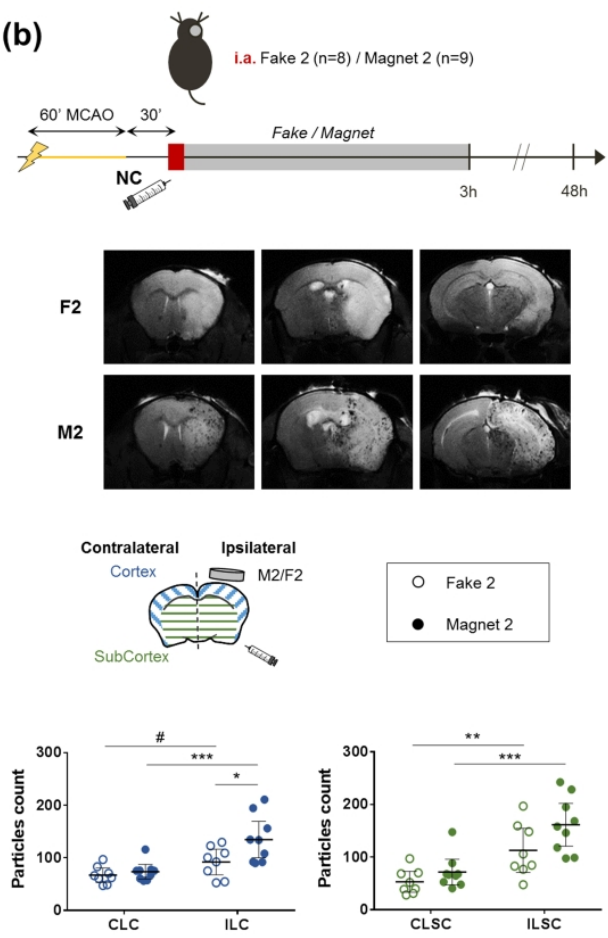

Figure 6. Biodistribution after MCAo and intraarterial-ICA NC administration showing the improved NC brain targeting with different magnetic devices and superior performance of the focused magnet design (M2). 

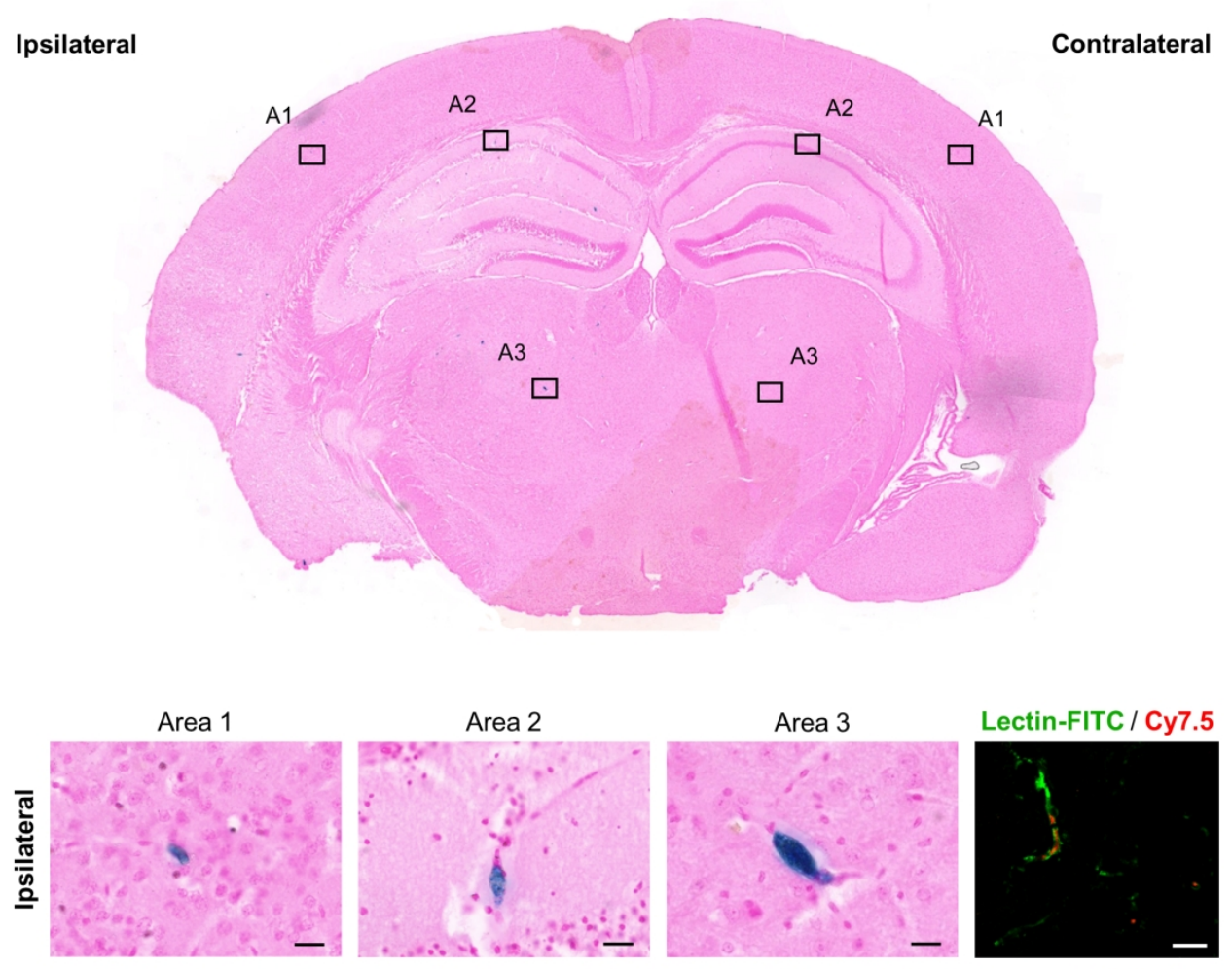

Lectin-FITC / Cy7.5
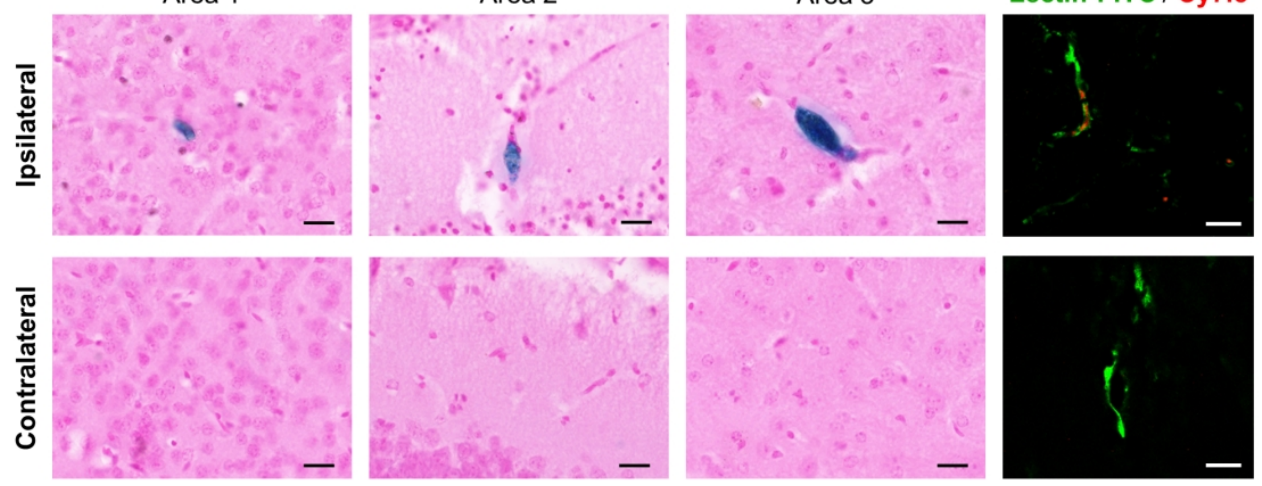

Figure 7. Verification of NC brain distribution by Prussian blue ferric iron staining (SPION) and fluorescence (Cy7.5).

$172 \times 172 \mathrm{~mm}(300 \times 300 \mathrm{DPI})$ 